\title{
Where are sunspots generated?
}

\author{
Evgeny V. Ivanov
}

IZMIRAN, 142190, Troitsk, Moscow Region, Russia email: solter@izmiran.troitsk.ru

\begin{abstract}
The data from the Greenwich Observatory for 1879-2003 (cycles 12-23) have been used to plot a time (Carrington rotations) vs. Carrington longitude diagram of distribution of the rotation-summed daily areas for each sunspot group. It has been revealed that most of the sunspots appear as clusters having common sources (Sunspot Formation Zones) that lie on a surface rotating with a period close to the Carrington rotation period $T=27.2753$ days. At the same time both the active longitudes and medium-size spots shift in the Carrington heliolongitude and rotate at an angular velocity corresponding to the rotation period $T \sim$ $26.8-26.9$ days. An attempt is made to explain contradictory data on the character of rotation of sunspots and active longitudes.
\end{abstract}

The data from the Greenwich Observatory for 1879-2003 (cycles 12-23) have been used to plot a time (Carrington rotations) vs. Carrington longitude diagram of distribution of the rotation-summed daily areas for each sunspot group. Such diagrams are illustrated in Fig. 1 for large long-lived sunspot groups with $S_{C R}>2000$ m.v.h. (a) and for mediumsize short-lived spots with 100 m.v.h. $<S_{C R}<2000$ m.v.h. (b). The examples are given for the north hemisphere and cycle 20. $S_{C R}$ is calculated for each sunspot group by summing up the daily areas of each sunspot group over a Carrington rotation. It has been revealed that virtually all sunspot formation zones (both for major centres of activity with the lifetimes up to 10 rotations or more and for small sunspots with the lifetimes of a few rotations) rotate at amazingly stable angular velocity corresponding to the Carrington period $T=27.2753$ days. It does not depend on either the phase of the cycle or the sunspot formation latitude. Besides, the width of these zones is rather small $\left(\sim 20^{\circ}-30^{\circ}\right)$ and changes little during their lifetime, independent of the phase of the solar cycle. It implies that sunspots are generated deep in the convection zone, where the differential rotation is virtually absent (Fig. 2a). Originating somewhere at the bottom of the convection zone, namely, in the layer with the angular velocity corresponding to the period $\mathrm{T}=27.2753$ days, the sunspots rapidly emerge to the solar surface for a time not exceeding 2-3 rotations. As a result, their shift due to the difference of the rotation velocities in the upper and lower layers of the convection zone is insignificant, and the width of the sunspot formation zone does not grow with time, remaining rather narrow.

Along with the rigid rotation of active longitudes, the sunspot formation zones corresponding to the medium-size spots (Fig. 1b) display another rotation mode with a rotation period $\mathrm{T}=26.8-26.9$ days (Bumba et al., 1991, 2000; Vasilyeva, 2002; Berdyugina et al., 2003) that results in their shift along the Carrington heliolongitude. This shift may be explained if we suppose that sunspots are generated in a layer rotating at Carrington velocity, but the generation is initiated by some external agent located in a lower and faster rotating layer. Such agent may be the poloidal magnetic field frozen in the return flow of the meridional circulation loop (from the poles to the equator) (Fig. 2b). Interaction between the poloidal magnetic field in the layer rotating with a period $\mathrm{T}=26.8-26.9$ days and the toroidal magnetic field in the tachocline (or near it) with the rotation period $\mathrm{T}=27.2753$ days may be what causes the shift of the sunspot formation zones along the Carrington longitude. The interaction is likely to occur in quasi-oscillation regime with the observed typical quasi-annual and quasi-biennial periods due to the 

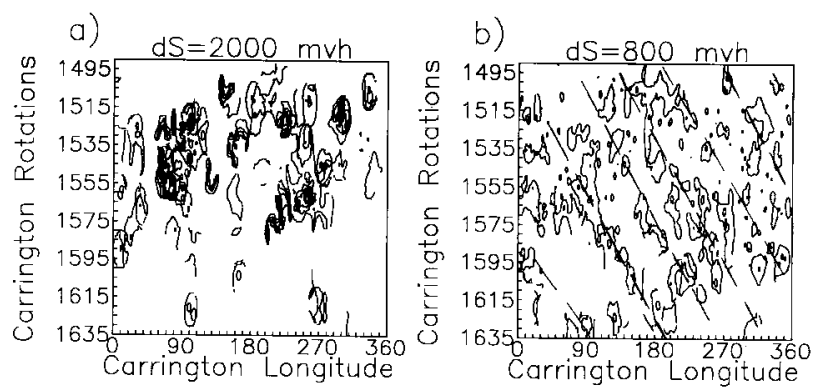

Figure 1. The time-longitude diagrams of the rotation-summed sunspot areas for major sunspots with $S_{C R}>2000$ m.v.h. (a) and small and middle sunspots with $100<S_{C R}<2000$ m.v.h. (b) in the northern hemisphere as observed in cycle 20 . The spacing between the contour lines is indicated at the top of the diagrams.
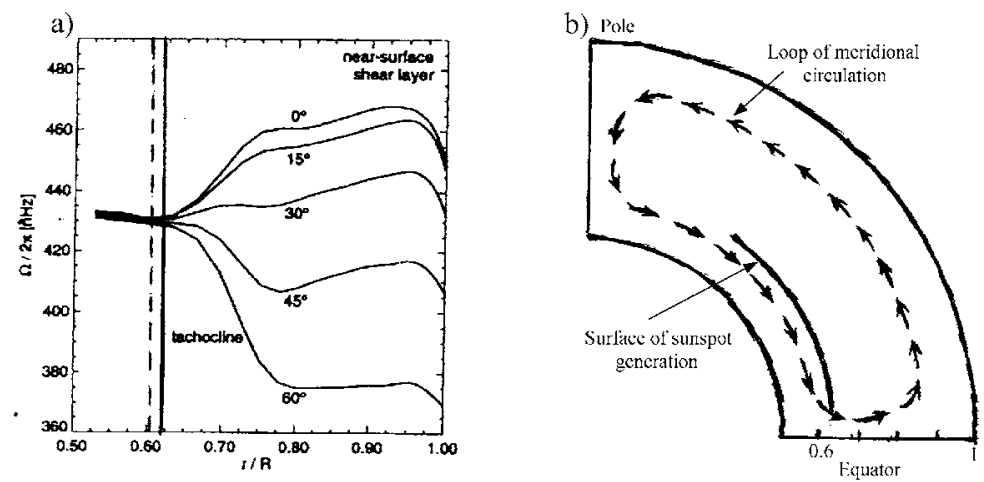

Figure 2. The location of sunspot formation zone (solid line) and return branch of the meridional circulation loop (dashed line) on the scheme, adapted from Howe at al (2000) (a) and on the scheme of convection zone (b).

non-axisymmetric solar oscillation modes. The moments of most intensive interaction of the two layers correspond to the occurrence of the most intensive sunspot formation zones. The same quasi-oscillation regime is likely to account for quasi-oscillations of the rotation velocity of the solar matter with the periods $\mathrm{T}=1.3-1.4$ years, which have been recently revealed in the tachocline region by helioseismologic methods (Howe et al., 2000).

\section{Acknowledgements}

The work was supported by the Russian Foundation for Basic Research (Projects N 04-02-16374).

\section{References}

Berdyugina, S. V. \& Usoskin, I. G. 2003 Astron. Astrophys., 405, 1121-1128.

Bumba, V. \& Hejna, L. 1991 Bull. Astron. Inst. Czechosl., 42, 76-85.

Bumba V., Garcia, A., \& Klvana, M. 2000 Solar Phys., 196, 403-419.

Howe, R et al. 2000 Science, 287, 2456-2460.

Vasilyeva, V. V. 2002 In Proc. Int. Conf. "Solar Activity and Cosmic Rays after the Reversal of the Polar Magnetic Field of the Sun", June 17-22, 2002, Pulkovo, St.-Peterburg, 95-100. 